\title{
但ie Bnftändigkeit in Straffadyen.
}

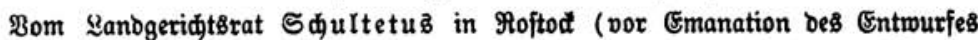

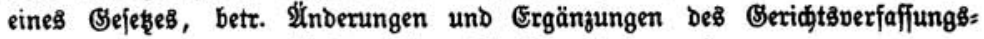
géę̧es und Der Strafprozęorbnung).

Unter ben gejezgeberifijen Fragen ber Begenwart fteht bie= jenige nach ber Einfübrung ber Berufung gegen bie urteile ber Straftammern augenblictlid wieber obenan und wiro vorausfidits

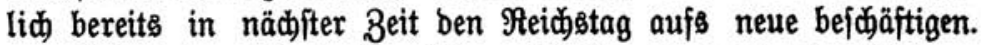

Bei biejem Stande ber Dinge bürfte es angezeigt erideinen,

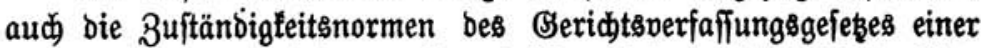
Prüfung zut unterzieben. Eine folde wirb $m$. E. auf bie Rot=

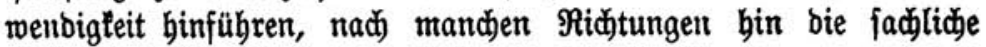
3uftänoigteit ber BSerid)te neu zu regeln, bamit aber bem einzufüh=

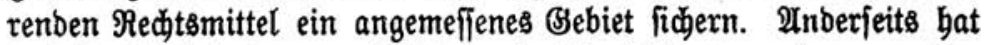
biejelbe insbejonbere aud bie materielle Bebeutung ber zur $\mathfrak{S}^{\mathrm{u}=}$ ftänbigteit ber Straftammern ftehenben Berbrechen und Bergehen zum Segenjtande und berührt baber bie Frage, ob biefelben, nadi= bem fie bas Ermittelungsverfalyren, eventuell bie \$orunterfuchung, bie befdließende uno endich bie urteilende Straftammer paffiert haben, troks Revifion und Wiederaufnahme bes ßerfabrens in ber That nody eite Nadiprüfung aud) ber Thatfrage burd) ein $\mathfrak{B e}$ rufungsggeridt erbeifdent.

Bei ber ftetig fteigenden $3 \mathfrak{a b l}$ ber Straffälle broht eine über= Iaftung ber Mittelgeridte, insbejondere burd fleine Strafiadjen,

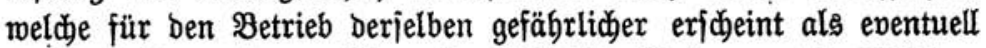
bie Zumeijung einer größern 2 nnzahl biejer Sadjen an bie Sdjöffen= geridbte. Daß̧ eine Belaftung Der Mittelgeridote mit fleinen Straf=

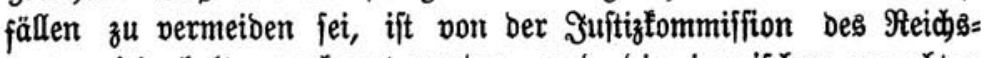
tags mieberholt anerfannt morben, und bie inzmifden gemadtet 
Erfahrungen baben bieje 2 nnabme beftätigt. Demu die Bearbeitung ber zahlreidhen Ileinen, meijt menigen Siategorieen angehörigen Straj=

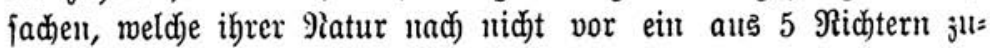

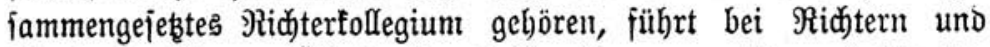
Staatsanmälten zu überoruß uno Ermübung, weldje auf bie $\mathfrak{B e}=$ bantlung berjelben, wie ber größern Sachen, nicht ohne jđäblid)en Einflü bleiben tam.

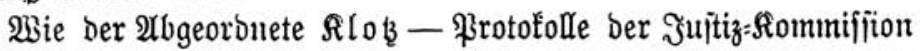
bes Reid)stages S. 604 - hervorbob, liegt "eine Entlaitung ber

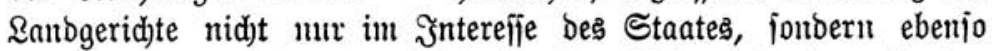

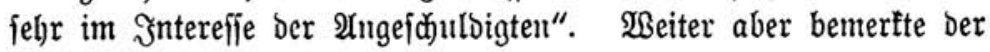

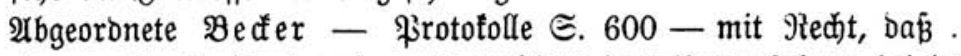
"Der große Rraftaujmano, mit welchem bas \$orverfal)ren bei bem

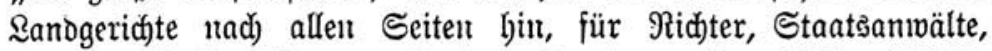

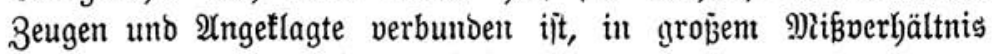
zu ben geringern Strajiachen fteht".

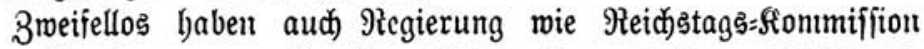
Diefent Ermägungen nach allen Seiten bin gerecht fu werben ge=

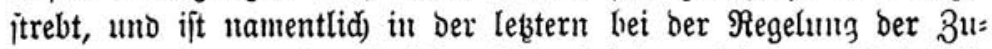
ftänbigfeitşgrenzen mit peinlid)er Ssentauigteit verfahrent. Dabei ijt übrigens mebrjac) barauf hingewiejen, baßs erft die Erfahrung lebren tönne, ob die 3ulfäntoigfeit ridutig bemefien jei, unt allfeitig

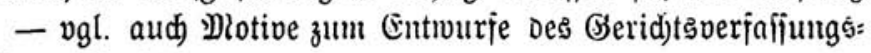
gejeß̧es ธ. 97 -

bie großße Edjwierigfeit einer ridjtigen Sompetenzregulierung anter: fannt.

Es iđheint mu, als menn Siebenten meijt theoretijd)er Satur in Der Jujtizfonmiifïion babiu gejül)rt baben, eimerjeits bie $3^{u}$ ftäubigłeit Der Scjöffengerid)te zu jel)r zu bej(bränten unt anter:

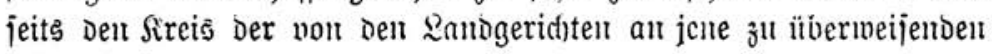
Eachent zu ielyr zu verentgen. Dbgleid) man, "nad)bem Die sBe: rujung an 5 ßid)ter sugelafjen worocn", fïr Die Ẽrweiterung oer Rompetenz Der E(h)öfiengerici)te plaioieren ju fömen glaubte,

\section{๘. $597-$}

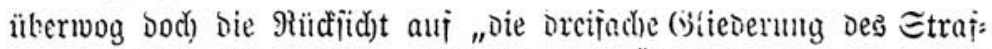
gejetsbuches in Nerbredjen, Wergebu mo lubertretungen" und liej

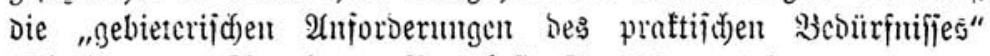

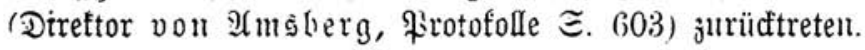


Währent ein Teil ber Rommififion ermog, „ob bas eimzelne Reat in feinen thatjäd)lidjen Momenten leidjt zu erfennen, obne Sd)mierigfeit unter bas Strafgeieb zu jubfumieren und beshalb ben Sdjoffengerichten zu ïbermeijen fei, - \$rototolle S. $205-$ murbe anderfeits hervorgeboben, baß "bie bödjite Madjt, meldje man ben Sdjoffen einräumen tönnte, eine ₹reikeitsentziebung bis zu brei Monater fei". So ift trok bes Bejdluffes ber Rommiffion: - Frotofolle S. 193 - „סas Snftem ber 3uftänbigfeitsfeft= ftellung fei nad) einzelnen Deliften, nid)t nach ber Strafböbe zu regeln" und troz̧ ber mannigfachiten Durdjbredjungen bes im \$ 14

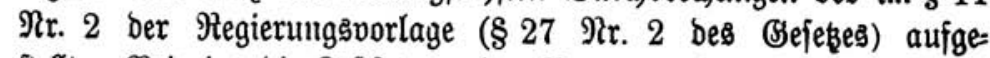

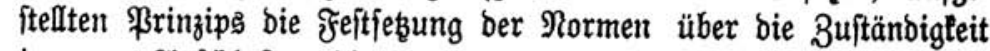

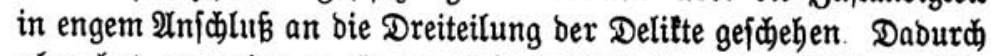

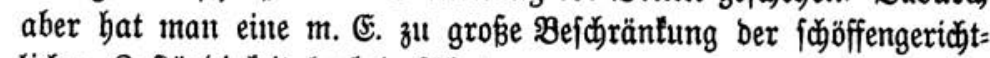
liđjen 3uftändigfeit herbeigeführt.

Demgegentïber wiro jeşt nad̆ gemad)ten Erfabrungen bie in ber Şuftiztommiffion vorbebaltene - vgl. \$rototolle S. $710-$

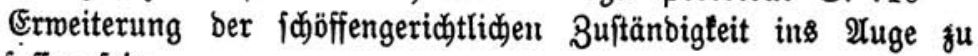
faffen fein.

(S)leidjzeitig aber wirb man zul ermägen baben, ob bie Er: fabrung nidjt aud) eine Befdränfung ber fdjwurgeridjtliden unb

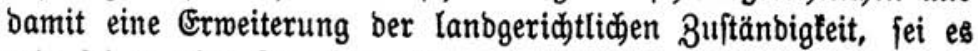
mit, fei es obne Sorreftionalifierung, geboten erjdeinen läpt.

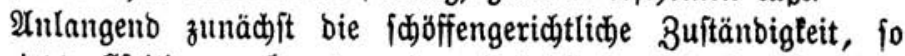

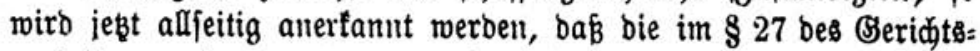
verfaffungggefę̧es unter 4-7 für bie bort genannten Eigentumbs:

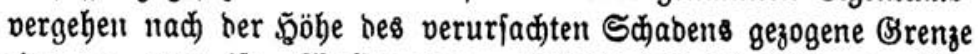
eine zu enge ijt. \$ollte man, obgleid) bas St.S.P. eine $\mathscr{U b}=$ ftufung ber (Eigentumbelifte nach) Der ફ口b̈he bes verurjachten

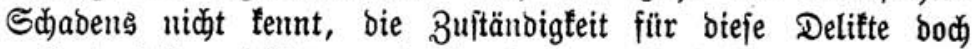
nach berjelben fefteken, fo burfte man berjelben unbebentlid) mit bem 2Xbgeorbueten $\mathfrak{R}$ räzzer - \$rototolle S. 323 - ben boppelten Vetrag ber vorbezeidneten Sd)abensfumme zul Srunde legen.

Die Erfahrung hat injwijd)en gelehrt, baß̉ bie gegen bie übertragung von Eigentumsbelitten auf bie Edjoffengerid)te er= hobenen Bebenfen umbegrïnbet find und bejtätigt, baß̧ gerabe bieje Delifte zu ben in thatjäd)lidjer uno red)tlidjer \$Beziebung einfachjten gehören. Sit bies aber ber Fall, fo wiro man ohne Bebenfent bie 
3uftändigleit ber Sđjoffengeridate für jene Delifte bis aulf eine Sd)abensfumme von menigftens $100 \mathrm{Mt}$. ermeitern bürfen (i. jeķt $\S 27 \mathfrak{N r}$. 8-10 und 13 bes (Fingangs gebacten (Entivurfä).

Rach) langiäbrigen Erfabrungen, weldje Berfaffer als Staats= anmalt zu machen S'elegenbeit gehabt hat, finb es veridjmindeno

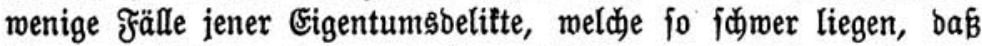
bei einem Schaben bes angegebenen Betrages eine böhere als eine 3 monatige Befängnisftrafe zu ermarten ftände. (5) miro vielmebr bie Zahl berjenigen Fälle, in welden troß̧ einer weit höbern

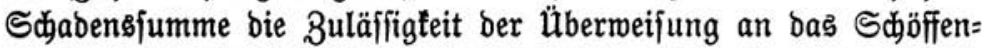
gericht unzmeifelhaft ift, eine groke fein. Man fann es beshalb gegenüber bem zu machenden grofen Beminn an Beit und Beld barauf antommen laffen, bas nach Bejeitigung ber Rautel bes $\S 16$ a bes Rommiffitonsentmurfes eriter Lefung bas Sdjöffen= gericht in einigen fälllen auf eine höhere als eine 3 monatige Bse= fängniş̄trafe zu ertennen haben wirb.

Beiter fommen biejenigen Delitte in Frage, für meldje, unter 3ulaffung ber überweifung an bie Sdöffengeridjte in ben meiften Fällen, bie 3uftänbigfeit ber Straftammern troz̧ ber mannigfachíten Einmendungen feftgejekt morben ift. Es find bies bie $\S \S 134$ (2)b= reißien ujw. öffentlicjer Befanntmachungen ujw. von Behörben; Beloftrafe bis zu 300 Dif. ober (Sejängnis bis zu 6 Monaten), 136 (Erbreçen u|m. amtlid, angelegter Siegel; Sefängnis bis zu 6 Monaten), 240 (Nötigung; (Sefäıgnis bis zu 1 Jahr ober (S)eld= itrafe bis zul 600 MR.), 241 (Bebrohung mit ber Begebung eines Berbredens; Befängnis bis zul 6 Monaten ober Belbitraje bis zul

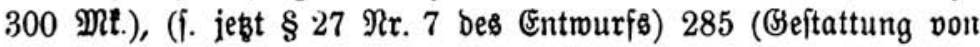

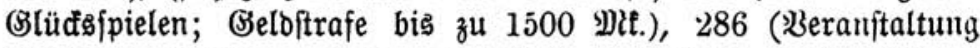
öffentlicher \&otterieen obne obrigfeitlidje Erlaubnis; उ̧efängnis bis zu 2 Jabren ober (Selditrafe bis zu 3000 Dit.), 291 (wiber= rechtliche 3ueignung veridoffener Munition; Befäugnis bis zu 1 Jahr ober Beldjtrafe bis zu $900 \mathrm{Mt}$.), 293 (unberedtigtes Jagen

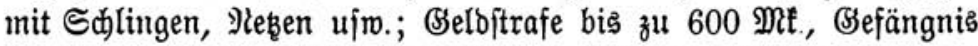

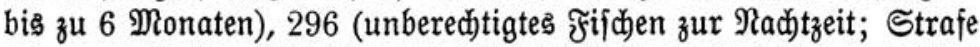
wie vorber) (zu §§ 291, 293, 296 St.(S.:B. i. jeşt Entmurf § 27 :ir. 11 u. 12).

Sinfictlich aller biejer Delifte hat fich bie Suftizfommifizion des Reic)stags rejp. bie von berjelben niebergejeşte Subfommiffion für bie übermeijung an bie Sdjöffengeridate ausgeiprod)en. 
(Es wirb audi) in ben Sreijen ber \$rattiter taum noch einem

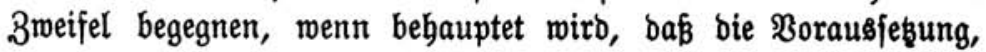
von weldyer jene Siommiffionen bei ihren Bejdlüffen ausigingen, leidjte Beftaltung jener Delifte in thatjädjlidjer und redytlider $\mathfrak{B}_{e}=$ ziehung, von ber Errfabrung für bie weitaus meijten frälle beftätigt

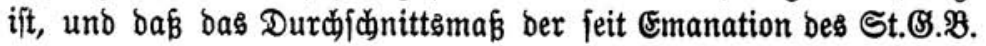
megen berjelben erfannten Strafen ein fo niebriges gewejen ijt, daßs basfelbe beutlich) auf bie füöffengeridjtlidje Buftänoigfeit hin= weift.

Der gejeşliche Thatbeftand ber meiften jener Delifte ift ein leidter, und nur in jebr feltenen ₹ällen mirb eine Berurteilung zu einer böbern Strafe als ber im $\S 27 \mathfrak{\Re r .} 2$ bes Beridtsoerfaffunģ= geję̧es feftgejeşten zu erwarten jein. Selbfit wo, wie etra im

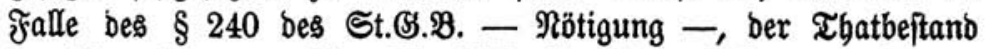
bes ఇergebens an fich nidht leidjt ift, find erfabrungsmäßig bie Eridjeinungsformen bes Delifts meift fo leidjte, baß nur gering= fügige Strafen erfannt merbent tönnen. Wie viele fleine Röti= gungen, burch weldje bei taum ernjtlicher, jebenfalls aber ungefäbr: liđjer Bebrohung ber Thäter eine für ihn faft belangloje \$̧anblung

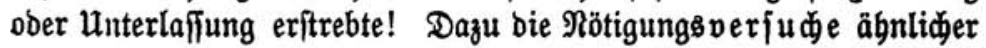

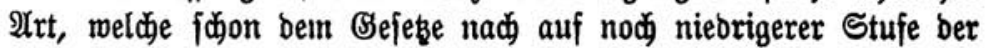
Strafbarfeit ftehen. Während feiner Thätigteit bei Seridten und Staatछanmaltiðaften ift bem Berfaffer nur ein einziger föwererer Fall von Rötigung betannt gemorben. In biejem wurbe megen Berleķung ber Sittlidłteit auf mehrmonatiges SSefängnis ertannt. Sonft regelmäpig jebr niebrige B̈elb= ober Befängnişftrafen. $\mathfrak{u}_{11}$ an foldhen Rötigungen uno Rötigungsperjuden fheitert in ben vielen Fällen, in welden biejelben mit zu überweifenden Sadjen fonfurrieren, bie übermeijung, ba nidht einmal bieje bei ibuen zu= läffig ift. Şier ift im Ŝntereffe ber Straftammern Mandel geboten uno ninbeftens bie 3ulaffung ber überweijung auszujprechen.

Uber jelbjt wenn für bie jämtlicjen in Rede ftehenden Delitte bie überweifung zugelaffen mürbe, bie lanogeridtlidje Sompetenz aber aufredt erbalten bliebe, mürbe ber zmects inrer 2lburteilung in

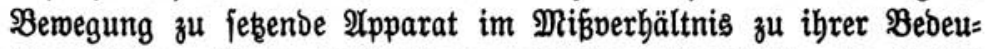
tung fteben. Diejer exjdeint es vielmebr entiprechend, fie einfach

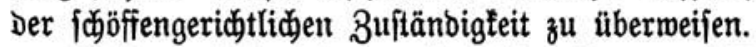

Anlangend insbejondere bas Bergeben aus $\S 286$ bes St.(3.P., jo ift anzuertennen, daß̉ bie Beurteilung des Thatbeitandes in that= 
fächlidjer, namentlid) aber in rechtlicher ßeziebung Sdjwierigteiten bieten fann. (E) mag aud hervorgeboben werbell, baß bas ßer=

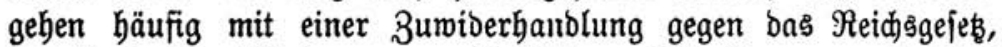

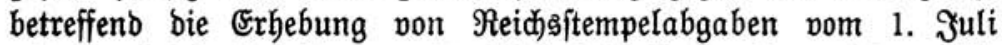
1881 und bie Novelle vom 29. Maa $188 j$ tonfurriert. Wenn fid bie Majorität Der Rommijijion aber troz nicht zu vertennender $\mathfrak{B}=$

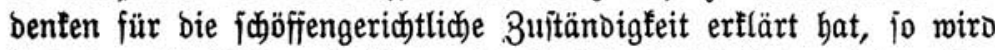
bies im Şinbliaf auf bie große Unbedeutendheit oer bei weitem meiften ₹̧älle bes Delitts gejojeben jein. Die Erfabrung aber hat bieje $\mathfrak{A n n a h m e ~ D u r c h a u s ~ b e f t a ̈ t i g t . ~ D i e ~} \mathfrak{A}$ usjpielung einzelner von fleinen ફ̧anbmerfern angefertigten Mobilien, beren $\mathfrak{A b j a z ̧}$ auf Dieje Weije berbeigefübrt werben foll, von $\mathbb{E}[=$ unb anbern geringmertigen

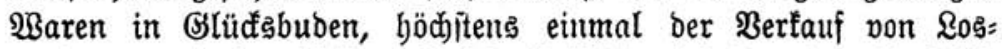

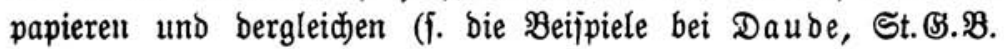

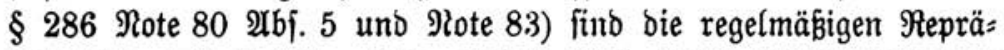
fentanten bes $\S 286$. Fil groß̧er Teil biejer Unternebmungen wirb fdjon um besmillen jelten einen größern Unifang anmebmen, weil biefelben wegen ber faum vermeiolidjen D̈ffentlidjeit zu febr ber Esefahr ber Entbectung ausgejekt find.

Anlangento aber bie Ronfurrenz bes $\mathfrak{B}$ ergehents mit Zumiber=

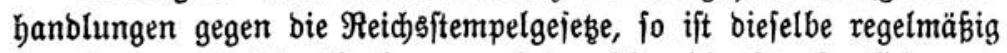
eine jogenannte Joealtonfurrenz, bei meldher bie Strafe allein aus § 286 bes St.ङ. $\Re$. zu beftimmen ift. Eventuell aber müroe gemäß

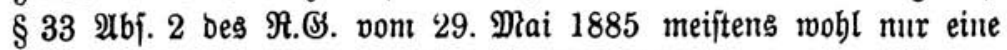
Dronungsftrafe von 3-30 $\mathfrak{M t}$. zul erfennent fein.

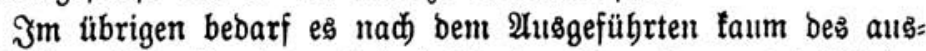

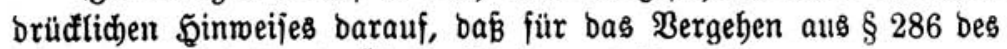
St.ङ.2. jebenjalls bie übermeijung zuzulajīen märe.

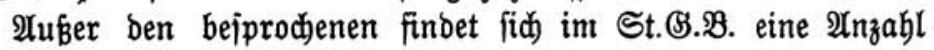

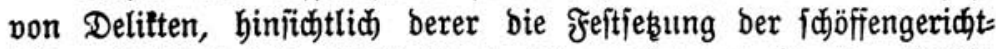
lidien Buftändigfeit gleidjfalls in Frage fommen fönnte. Dabin gebören bie Fälle ber $\S \S 123$ $2 b j .2$ (bemaffneter uno gemeinjidaft=

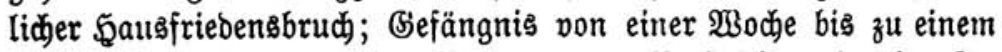

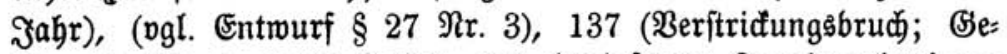
fängnis bis zu einem Jabr), 290 (unbefugtess Эngebraudunebmen verpfänbeter Begenftänbe burch öffentlic)e Pfanoleiher; (Sefängnis bis zu einem Эabr, baneben - fafultativ - SSelditrafe bis zu 900 Mft.), 298 (Entlaujen mit ber நूeuer; B̉efängnis bis zu einem

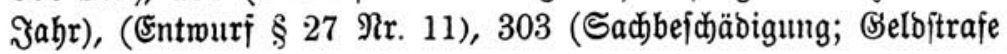




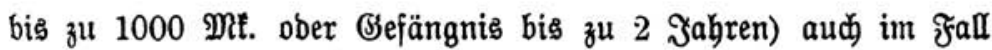

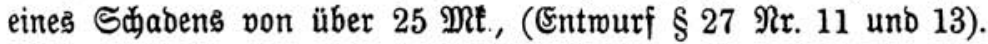

Die $\S \S 137,290,303$ hatte bie Subfommiffion ber Jujtiz= fommififion gemäßs bem 2 Untrage bes $\mathfrak{A b g e o r o n e t e n ~}$

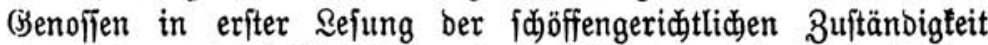

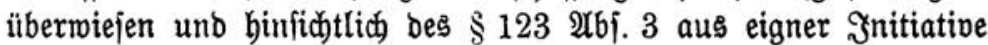
Daşjelbe gethan. Die Jujtiztommijifion hat bie Rompetenz ber Straffammer für bieje Bergeben mieberhergejtellt.

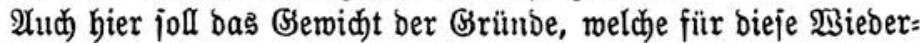

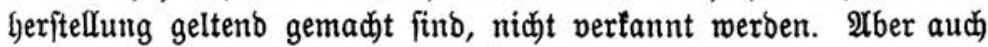
bier muß anberjeits auf bie Erfahrung hingemiejen merben, $\delta a \dot{B}$ verhältnismäß̧ig mentig Fälle ber fragliçen Bergeben einen idwerern Eharafter baben. Selbit ber qualipizierte Қausfriebens:

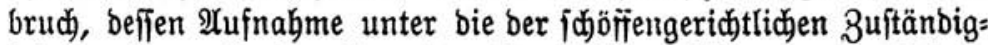
teit zu ïbermeifenden Bergeben bie Subfommiffion ber Suftiztom= mififion in zmeiter Rejung einfitimmig, bie Sommiffion jelbit mit Stimmenmebrbeit abgelehnt hat, — \$rototolle S. 709 und 711 tann als ein fo "tompliziertes" Bergehen nidjt angejehen werben, daß für basfelbe als Regel bie Rompetenz ber Straftammer feit= zubalten wäre. Die Dualifilationsmomente ber Bemaffnung und ber (Semeinfdjaftlidjeit ftellen fid) jehr häufig nidht als jo jömer= wiegende bar, baß ber Thatbeftand bes ßergebens fid) baburd er=

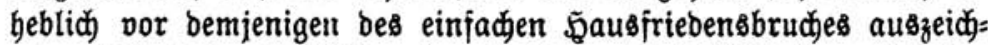
nete. Dit find es Stöcfe ober ähnlide, hinijdytlich ihrer Bsejähr.

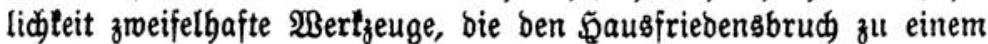
bewaffneten machen. Das Qualifitationsmoment ber Bemeinfdajt= lidjeit aber miro nidjt felten burd einige menige \$erjonen ber= geftellt, bei beren 3ujammenfübrung ber 3ufall eine mebr ober minber bebeutende Rolle gejpielt bat.

Wie maı aber aud) über bie regelmäßige Zuitänbigteit jür die fragliden Delifte benfen mag, die überweifungşähigfeit wiro man feinem berjelben abiprechen bürfen und beşbalb aud bem ș 290 (Ĵngebraudunabme verpfänbeter Sachen burd) ßfandleiher) zujpreçen müfjen.

Warum gerabe biefes Nergeben von ber übermeijung aus: gejd)loffen ijt, obgleid) basfelbe, wie man meinen jolle, feinem Thatbejtande nach bejonbers für biejelbe geeignet erjojeint und Protofolle S. 709 - nod) in zmeiter Rejung von ber Subfom= mijfion für bie jđjöffengerid)tliche 3 ujtänbigfeit geeignet befunden 
war, ift nidyt erfennbar. Man müß̈te bemielben benn wegen ber zum Thatbeftanbe gehörigen Dffentlidfeit bes \$fandleiggemerbes ben Eharalter einer gewiffen Bemeingefäbrlidleit beilegen wollen. Enblich find einige Delitte bervorzubeben, für meldhe bie

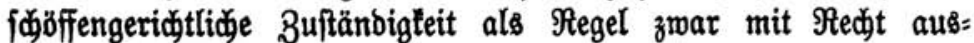
gejdlofien ift, weldhe m. E. aber als für bie übermeifung geeignet

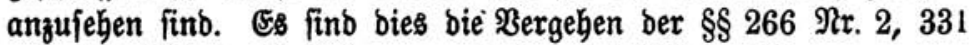
uub 347 $26 j$. 2., (vgl. bagegen Entwurf $\S 75 \mathfrak{R r}$. 11).

Sif man bei ben Beftimmungen über bie überweijung mit

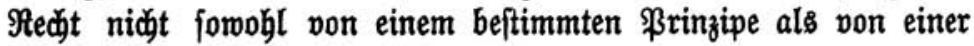

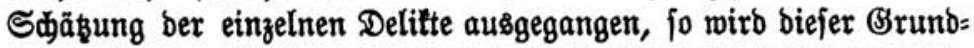
faßs und eine ridtige Bürbigung ihrer ftrafredtlidjen \$ebeutung aud bei biejen ßergehen bie Bulaffung ber überweifung angemefien eridjeinen Iafien.

Die 3iffer 2 bes $\S 266$ bes St.(S..8. ift allmählid für Staats: anwaltidaften unb Bseridjte zu einer crux gemorben.

Seitbem ber Begriff bes "Bevollmädjtigten" mebr und mebr erweitert worben ift, hat fid bie Babl ber unter ben cit. $\$ 266$ fallenden und beshalb von ben Straffammern abzuurteilenden fleinen Unterjolagungen, weldje von oft febr zweifelbaften $\mathfrak{B e v o l l = ~}$ mädobtigten verübt fino, ftetig vermebrt. Rellner, \&ehrlinge uno Ģanolungsireije ujw., weldje bie unbebeutendften Unterjळlagungen veribt haben, müffen wegen ibrer fragwürbigen Bevollmädtigten= qualität vor bie Straftammer.

Benn aber aus ben ftatiftifjen überfichten zufammengeftellt warbe, meldje Strafen feit Emanation bes Strafgeję̧budes megen

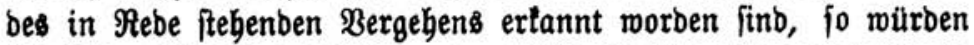

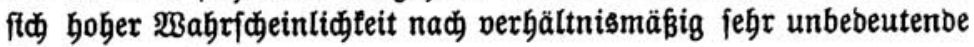
Strafen ergeben.

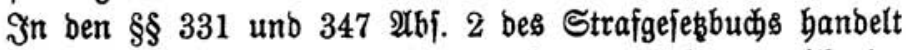
es fich zmar um ßergehen im 2 mte, aber um foldje, meldye im Şödjftmaß̧e mit Bselojtrafe bis zu 300 Mt. ober Befängnis bis zu 6 Ponaten rejp. mit \&̈efängniš bis zu 6 Monaten ober Sielditraje bis zu 600 Mt. bebroht find.

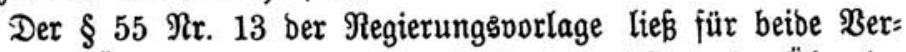
geben bie 亿̇berweijung zu. Die Juftiz=Siommiffiton, in überein= ftimmung mit ihrer Subtommiffion, hat biefelbe in Megfall ge= bracht. 2rber aud binjidytlid biefer Strafbeftimmungen fprechent bie vom Bejes angebrohten niebrigen Strafen, bie Einfachbeit ber 


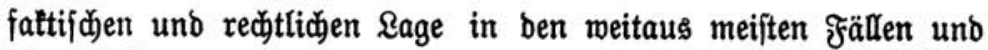
bie erfahrungsmäß̈ig erfannten niedrigen Strafen für bie îtber= weijung.

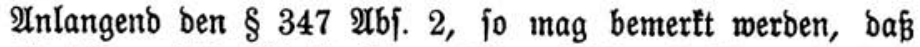

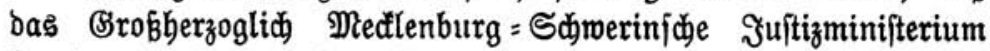

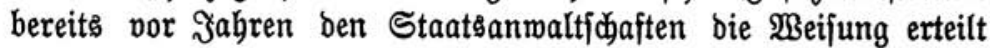

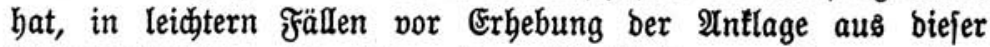
Strafbeftimmung bie 2ftten bem Minifterio vorzulegen. Den (Srund biejer 2̂noronung wirb man in ber Erfabrung fuchen bürfen, baß̧ fehr viele unter jene Beftimmung fallenbe Berfehlungen fo unbebeutenber $\Re a t u r$ fino, baß fie geeigneter für ein bisziplinares als für ein friminelles ßerfahren erfdeinen uno biejes obne benten niebergejकlagen merben tann.

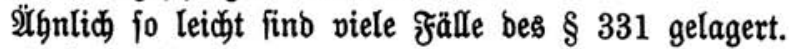

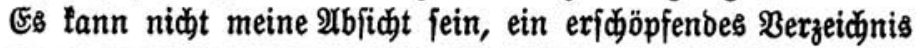
berjenigen Bergehen aufzulftellen, für weldhe bie Feffieģung ber

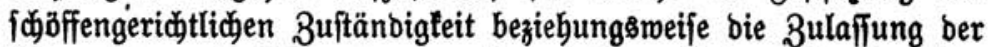
übermeifung in frage tommen tann. Se nad̆ bem Stanbpuntte, ben man zu ber Bebeutung ber in Betradst tommenben Delitte einnimmt, würbe ein foldeses entweber als lúctentyaft ober als zu viel umfafieno erfajeinen.

Wie verfajieben ber Stanbpuntt in biejer 2 eziegung ift, be= weift, um nur eins hervorzubeben, ber $\mathfrak{u}$ mitano, baß

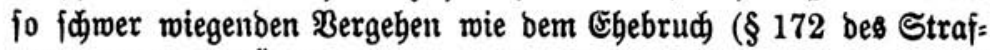
gefesbucks) bie ltherweijung zugelaffent ift. So mentig zmeifellgaft es $m$. E. fein tann, baß biefelbe zu befeitigen ift, fo unbebentlich erfdeint biefelbe für mandje ber voraufgefübrten Bergeben, beren itrafrechtliche \$ebeutung entfernt nicht an biejenige bes Egebrudes

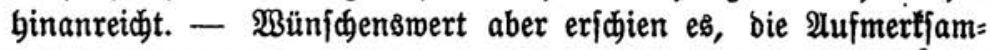
feit ber maßggebenden Fattoren auf bie Irbänberungsbebürftigteit Der 3uftändigteitsnormen binzulenten.

Eine $\mathfrak{2}$ inderung berjelben würoe $m$. E. vorzagşweife in ber Ermeiterung ber fdö̈ffengeridjtlichen Sompetenz uno bes SReifes Der zu überweijendent ßergeben zu fudjen fein.

Ĵn erfterer \$eziebung bürfte insbefortbere eine erheblide Ex:

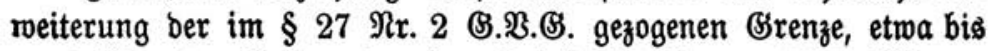
zu 6 Monaten Bsefängnis ober Beldftrafe bis zu 1500 Mt., zu erftreben fein. Nach ben inzwifiden gemacten Erfabrungen ift

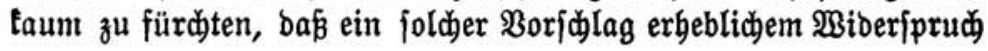


begegnen wirb, wie er ihn bei ber Beratung bes Berid̄tônerfaffungs= gejeß̧es zmeifellos hernorgerufen haben mürbe (vgl. aber $\$ 27$ ग?r. 2. bes (Entmurfes). Denn thatiädlich ift es längit babin gefommen, daß ben Schöffengeridjten viele Strafjachen überwiefen merben, in benen biefelben zmeifellos auf eine jebr viel höhere als eine brei= monatige Bsefängniş̄trafe zł erfennen haben.

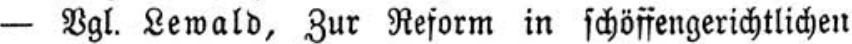

Strafiadjen in Z 101 fi. -

Rach) glaubmürbigen Mitteilungen trägt eines ber größ̈tent Sanogeridute fein Bebenten, in allen Fällen, in melchen "nach Dent

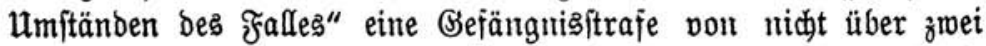
Jahren zu erwarten ift, bie übermeijung auszujpred)et. Dieje

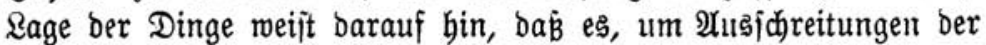
Praris zul begegnen, wünichensmert ijt, auj Dem ßege ber Bejets= gebung bie von Dem Bleridjtsperfaffungsgeję̧e gezogenen Bsrenzent zu ermeitern.

Unlangent bie übermeijung, jo rürbe es mir, entiprectento ber vorgejd)lagenen Ermeiterung Der jüb̈ffengerid)tlidjen Fompetenz, angemeffen erjojeinen, biejelbe bei benjenigen Bergeben zuzulaffen, roelde mit Bsefängniştrafe von hödjitens 1 Jahr oder Bselojtraje von hödjften 3000 IMf. bebroht j̈̈t, eine überweijung weiterer einzelner Fälle vorbehalten (vgl. aber Entmuri § 75 Nr. 11).

Dabei wäre zu ermägen, ob bie fäj̈ffengeridjtlidje Straj: befugnis auf ein beftimmtes Mlaßs, etma 6 Plonate Bejängniş ober Beloftrafe bis zu 1500 Mf., zu bejdränten und eventuell bie $\mathfrak{B e r =}$ pflidutung bes ङerichts zur 3urüđtmeijung an bie Straffammer aus: zuipredien jei.

\section{- Bgl. Semalo a. D. S. 104. -}

Bei einer bementiprechenten $\mathfrak{A}$ uglgleichung ber 3uittänoigfeits: normen für Straffammern und Sdjöffengerichte ijt zu erhoffen, baß̧ fomohl die von jenen als bie von diejen abzunteilenben Straj: faden beffer zul igrem Rechte fommen als biaher, insbejondere

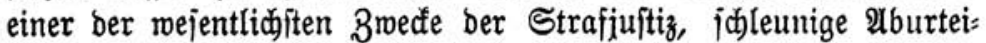
lung ber Strafthaten, geförbert merbe.

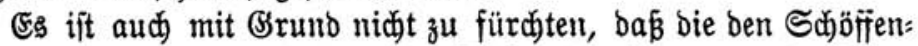
geridjten meiter zuzumeifenden Strafjachen burch bas vor benjelben ftattfindende fürzere Berfahren in ihrer Behandlung beeinträchtigt merben. Denn es ift allmählich mohl bie überzeugung burdigge: brungen, baß̧ bie im Şnterefife ber Ingeflagten für bas ミerfahren 
vor ben Straffammern eingejührten ミicherungsmaßaregeln —§ 199 ! (vgl. aber Entmuri \$ 199) - in mannigjuchen Beziebungen über

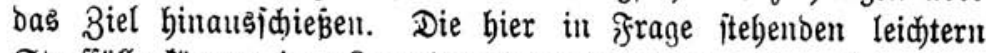
Straffälle tönnen jene Rautelen ohne Sd)mälerumg ibrer \$ebeutung jebr mohl entbebren, ulo mürben in ifrer grösern 3 abl felbit jür

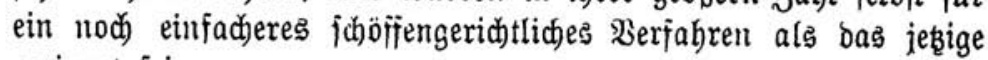
geeignet fein.

- Bgl. Remald a. D. -

Wie eine Ermeiterung Der \{dö̈j̈engeridtliden Rompetenz, jo

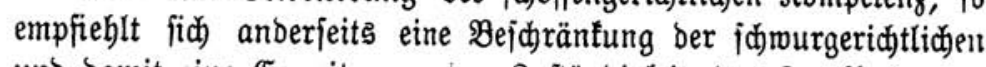
uno bamit eine Ermeiterung Der Buitänbigłeit ber Straftammern. Wenn man bafür eintritt, jo befunbet bies feinesmegs prinzipielle

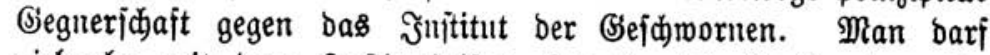
vielmebr mit bem Juftizminifter \&eonharbt (2. Beratung im

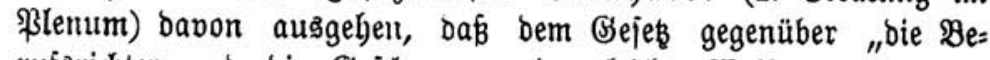

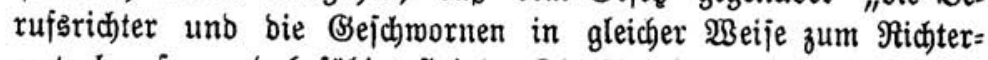
amte berufen unb befäbigt find". Die Unerfennung biejes Saß̧es barf aber nidjt zu eimer überijojreitung ber ber fdjwurgeridjtlidjen Sompetenz naturgemäß gefitedten Brenzen fübrelt. Die Sd̆wur=

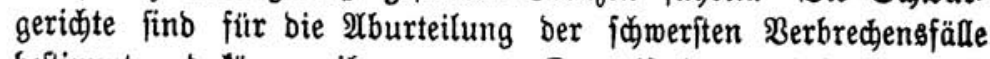
beftimmt und tönnen ifrer ganzen Drganifation nach füglid audi) nur bafür beitimmt fein. (Es bürien ihnen Deshalb feine $\mathfrak{B}$ er=

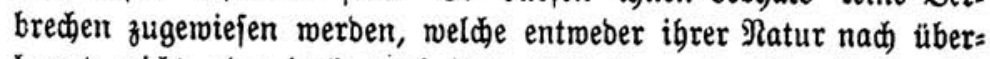
baupt nidit ober boch nach ibrer (seitaltung in Finzeljalle nidjt babin zu rechnen fino.

Bon biejem Standpuntte aus ijt es als eine mejentlicke $\mathfrak{B e r}=$

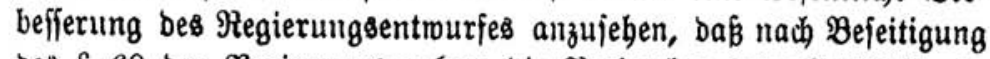

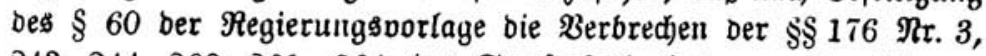
$243,244,260,261,264$ bes Strafgeję̧budbes von ber finrwur= geriditlidien Sompetenz ausgejd)lofien find. (F) giebt aber auker

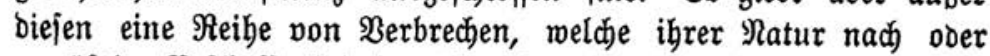

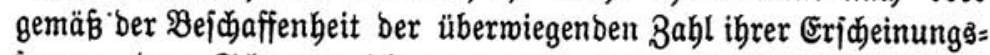
formen bem Sdjwurgerichte entweber überhaupt nicht überwiefen oder burd જeibehaltung ber Rorrettionalifierung für leidjtere fälle bätten entzogen werben jollen.

Dahin gehört in eriter Sinie ber $\S 118$ bes Strafgejeł̧buches. Ess ift zuzugeben, baß̧ mandje Fälle biejes ßerbredjens fo jomere

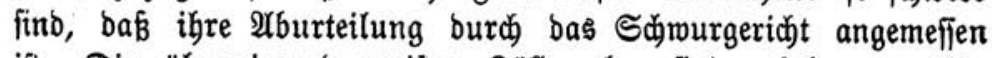
ift. Die übermiegend meijten ₹älle aber fino erfahrungsmäß̈g 


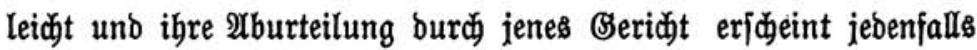
entbehrlicid.

Die Sörperverleķungen, weld̄e bas ßerbrechen zu einem fupweren ftempeln, find oft fo unbebeutende, mannigfad burd bas 2Uuftreten ber Forftbeamten mitveranla Forftfrevlern ujw. in ber erften Erregung bei ifrer Ergreifung ver= uriacht, baß bie bei Bubilligung mildernber $\mathfrak{U}$ mitänbe zu erfennende Strafe bas angebrobte Strafminimum meift nidjt erbeblid über= fidreiten wiro.

Will man fid baber nidat entid̄ließen, den $\S 118$ überbaupt ben Straftammern zuzumeifen, fo wäre $m$. (E. Doch - unter

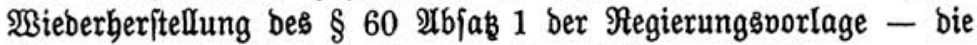
übermeifung an bie Straftammer für zuläffig zu erflären.

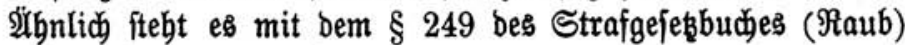
(ję̧t Entmurf § 199).

Sier, wie beim $\S 118$, weift bie 3ulafiung milbernber $\mathfrak{u} \mathfrak{m}=$ ftänbe und bas bei Zubilligung berfelben angebrohte Strafmaß barauf hin, baß Fälle leidjterer 2 rt vorgejeben find, weldhe bie Er= fennung niebrigerer Strafen notmendig maden. - Die Erfabrung hat bieje Innahme beftätigt.

Die Dehnbarteit bes Begriffes ber "Bemalt" ift bie uriache,

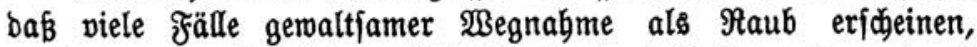
weldje von ber Brunbform biejes ßerbrechens („Entwentung burd)

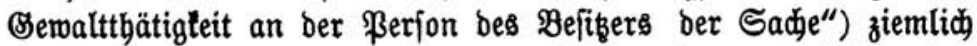
meit entfernt fint. Die Bemalt ift oft eine fo unerbeblide, bas

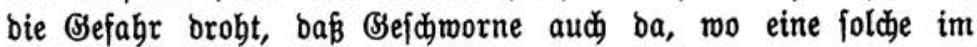
Redtsfinne vorliegt, wegen biefer ibrer Unerbeblichleit freifpredjen werben. - Man bente weiter an bie Fälle, in welchen jemand, um fïh für eine frorberung bezahlt zu machen, feinem Sdjuldner gemaltjam eine Sacje regnimmt, Fälle, melche nach ber jubjeltiven Seite fehr leidjt zu liegen pflegen $\mathfrak{u}$. a. m., um fid zu überzeugen, baß ber $\Re a u b$ in vielen feiner Erfideinungsformen nicht vor bas Sdymurgeridt gehört.

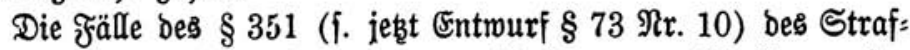

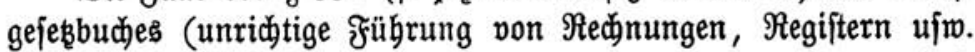

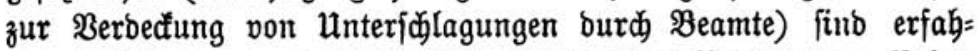

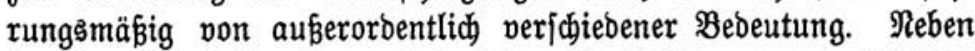
ben tomplizierteiten, lange fortgejeşten ßerfäljobungen von Büchern

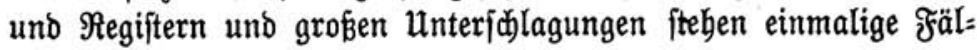




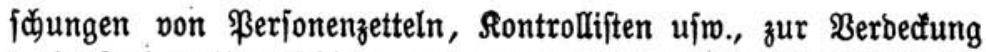

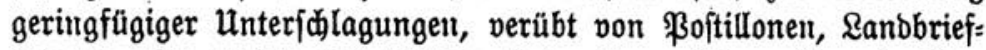
trảgern ujw. F̧älle ber leştern $\mathfrak{U r t}$, für weldje milbernoe $\mathfrak{u} \mathfrak{m}=$ ftänoe und ein Strajninimum von 6 Monaten B̧efängnis zu= gelaffen find, entiprechen wieberum nidht ber Bebeutung bes Schwur= geridts. Nod) ein antrer şrund aber als bie nidjt erbeblide Bebeutung vieler Fälle bes § 351 weift barauf bin, biefelben ber 3uftändigfeit bes Sdimurgeridts ganz ober burd bie Rorrettio= nalifierung teilmeife zu entżiehen. Das ift bie Sdimierigleit ber

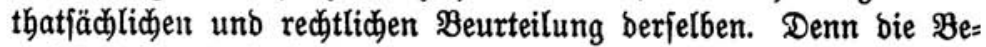
weisfübrung bregt fich in benfelben oft um fobper erfennbare, exit

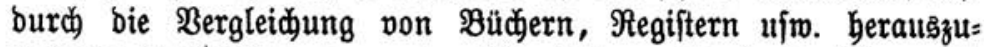
ftellende Berfäliøungen, uno nidat felten wirb in berjelben mit einem umfangreiden urtundliden Material operiert. IInberfeits

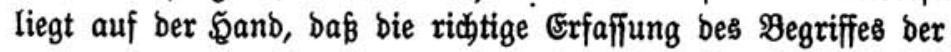

„8̧ur Eintragung Doer Sontrolle ber Einnabmen ober $\mathfrak{2} u \varepsilon=$ gaben beftimmten Redinungen" uf w.

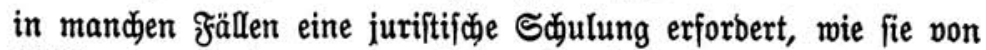
Befकwornen weber vorausgejeşt nod geforbert werben tann.

Bei ber Peantwortung ber in Betradjt fommenten Fragen werben bie Beffimornen baber mehr nod als jonit auf bie thnen von bem Borfizenden bes Sjeridsts unb bem Staatsanwalte zu teil werbenbe Belebrung angerwiefent fein und leidht auf ein felbfänöbiges $\mathfrak{u} r t e i l$ verzichten. Daraus aber ergibt fïh) eine bie Selbftänbigleit

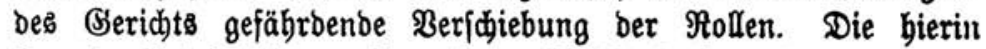
liegende Befahr ift gerwiß größer als bie Sntonvenienz, baß ein

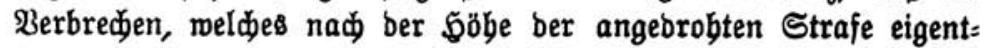
lich vor bie Bejfimornen gebört, entweber ganz ober in geeigneten Fällen ben Straftammern zugewiefen wiro.

Dies als ridtig zugegeben, wiro man obne große \$ebenten auch Die ßerbrecten ber $\S \S 209$ und 212 Der Ronturborbnung (betrügerifcher Banferott rejp. Begünftigung eines foldhen) ber fd̈wurgeridytlidjen Rompetenz ganz ober teilweife entżieben bürfen (f. jeşt Entwurf § 73 शr. 11).

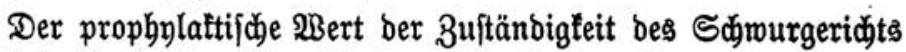
für biefe Berbredjen ift nidyt fo groß als bas Intereffe an einer gründlichen uno gerechten 2 tburteilung berfelben. Sierfür bieten aber aud in biejem Falle bie Straffammern bie größere \&arantie. 
Denn faít fämtlidje Fälle ber $§ \S 209,212$ ber Sonfurs=:Dro= nung erforbern eine umfangreidje Bemeiserbebung burch urfunben, (seidjäftebücher ujw., melder bie (sejdjwornen jelbit bei ber größten 2Uufmerffamfeit nur teilweife zu folgen imitanbe fein werben, unb beren Ergebnis ridjtig zu beurteilen fie baber vielfach nidjt in ber Sage finb. Dazu fommen Shmierigfeiten rechtlidjer 2 (rt, weldje

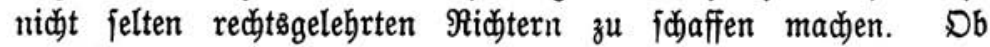

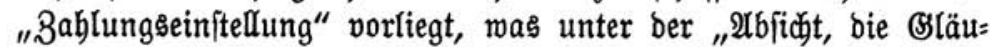
biger zu benachteiligen", was unter ber "श्2uffitellung eines erbid)=

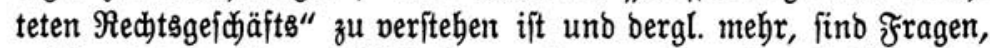
weld̄e einigermaßen felbftänbig zu beantworten ben Bsejdimornen oft iaum möglich fein miro. Bemeiserbebung, ßlaiboyers uno Rechtebelebrung neל̧men in foldjen Fällen bie toftipielige Zeit bes

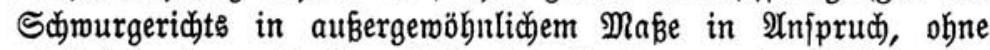
vielfach) zu bem eritrebten Biele, ber Seminmung eines ficjern $\mathfrak{u} r=$ teils auf feiten ber Ssefdrmornen, zu fübren.

Im Interefie ber Selbitändigfeit und bes 2 Injehens ber Juftiz ijt baber eine $\mathfrak{A} u s b e j$ jeibung folcher und ähnlidjer Fälle von ber (a)murgericjtlichen Rompetenz und bie 3umeijung berfelben an bie Straftammern wünidensmert. Db bies allgemein ober im $23 e g e$ ber Sorreftionalifierung gefajiebt, ifit nidjt von wejentlicher $\mathfrak{B e}=$ beutung.

Werben bie Straftammern in ber vorbejadriebenen $\mathfrak{A} r t$ von fleinern Straffachen entlajtet unb baburd in ben Stand geję̧t, fich ben größern mit gauzer Sirajt zu mibmen, jo erjobeinen fie

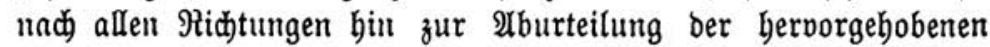
Berbrechen geeigneter als bie Sd)wurgeridjte, welche, von anberm abgejeben, babei zu fehr auf bas technif(d)=juriftifd)e (sebiet geführt merben und bort leidbt verirren fömnen.

Mögen nun bie auf bie Einfübrung ber Berufung gegen bie Urteile ber Straftammern gerid)teten Beftrebungen Erfolg haben

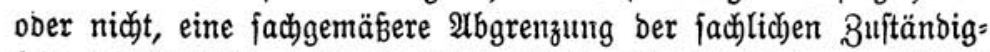
feit ber (S)eridate wirb in jebem Falle eritrebensmert, in eriterm aber angemeffien mit ber Finfübrung bes neuen Rechtsmittels zu verbinben jein. 